\title{
A Channelized Digital Ultrawideband Receiver
}

\author{
Won Namgoong, Member, IEEE
}

\begin{abstract}
A channelized digital ultrawideband (UWB) receiver that efficiently samples the UWB signal at a fraction of the chip frequency is proposed. The received signal is channelized in the frequency domain by employing a bank of mixers and low-pass filters. After sampling at a much reduced frequency, digital synthesis filters optimally estimate the transmitted signals. The signal-to-noise ratio (SNR) of the proposed receiver has been solved and compared against an ideal conventional receiver, which is defined as a receiver that samples at the signal Nyquist rate. When finite resolution analog-to-digital converters (ADC) are employed in the presence of a large narrowband interferer, the proposed receiver significantly outperforms the ideal conventional receiver. For example, the SNR of the proposed receiver is as much as $20 \mathrm{~dB}$ higher than the ideal conventional receiver when a 4-bit ADC is used in the presence of a $50 \mathrm{~dB}$ (relative to the noise floor) brickwall narrowband interferer with a bandwidth of $15 \%$ of the chip frequency.
\end{abstract}

Index Terms-Broadband communication, filter bank, receivers, spread-spectrum communication, ultrawideband (UWB) communication.

\section{INTRODUCTION}

$\mathbf{T}$ HE ultrawideband (UWB) radio is a relatively new technology that is being pursued for both commercial and military purposes. The rationale for deploying UWB radio systems lies in the benefits of exceptionally wide bandwidths in very low frequency ranges, thereby achieving a combination of very fine time/range resolution along with the favorable propagation condition of material penetration at low frequencies [1]. Some potential applications for UWB include identify-friend-or-foe (IFF) systems, covert communications, RF tagging, wireless LAN systems, etc. [2].

The UWB radio operates by spreading the energy of the radio signal very thinly from near d.c. to a few gigahertz. Since this frequency range is highly populated, the UWB radio must contend with a variety of interfering signals, and it must not interfere with narrowband radio systems operating in dedicated bands. These requirements necessitate the use of spread-spectrum techniques. Both time-hoping spread-spectrum (referred to as impulse radio) [3], [4] and direct-sequence spread-spectrum (DSSS) [5] UWB systems have been studied. This paper focuses on DSSS UWB systems, although many of the reception techniques described in this paper are equally applicable to impulse radios.

Although great headway has recently been made in efficient implementation of DSSS receivers, the UWB signal has funda-

Manuscript received May 17, 2001; revised September 21, 2001 and December 6, 2001; accepted December 11, 2001. The editor coordinating the review of this paper and approving it for publication is A. Svensson. This work was supported in part by the Army Research Office under Contract DAAD19-01-1-0477.

The author is with the Department of Electrical Engineering-Systems, University of Southern California, Los Angeles, CA 90089-2562 USA (e-mail: namgoong@usc.edu).

Digital Object Identifier 10.1109/TWC.2003.811177

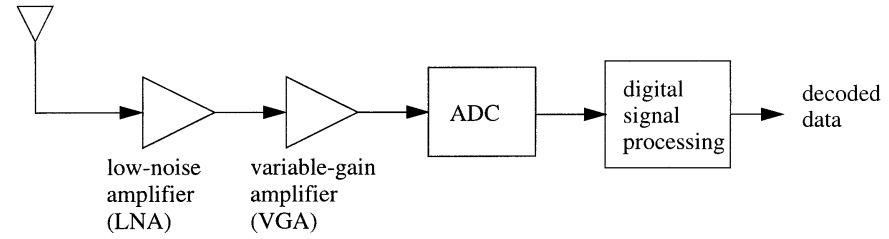

Fig. 1. UWB receiver architecture.

mentally different signal characteristics. Unlike existing DSSS receivers, the signal bandwidth of the UWB radio is at least an order of magnitude greater. Furthermore, the UWB radio must coexist with many other narrowband systems transmitting and receiving in the same bandwidth. Consequently, an UWB receiver has intrinsically different design requirements, which implies reception techniques that are also substantially different.

In an UWB receiver, the analog-to-digital converter (ADC) can be moved almost up to the antenna as shown in Fig. 1. Critical to this design approach, however, is the ability of the ADC to continuously sample and digitize at least at the signal Nyquist rate of several gigahertz. In addition to the extremely high sampling frequency, the ADC must support a very large dynamic range ( $>10$ bits) to resolve the signal from the strong narrowband interferers. Currently, such ADCs are far from being practical. The fastest reported ADC in CMOS process technology achieves only 6 bits of resolution at 1.3 GSamples/s [6]. ${ }^{1}$

As a result, existing UWB receivers perform receiver functions such as correlation in the analog domain before digitizing at a much reduced sampling frequency. Such analog receivers are less flexible and suffer from circuit mismatches and other nonidealities. These circuit nonidealities limit the number of correlators that can be practically realized on an integrated circuit (IC) to generally less than ten. Since over a hundred correlators may be required to exploit the diversity available in an UWB system [1], existing analog receivers suffer from significant performance loss. The analog circuit nonidealities also preclude the use of sophisticated narrowband interference suppression techniques, which can greatly improve the receiver performance in environments with large narrowband interferers such as in UWB systems. Consequently, to achieve high reception performance, the UWB signal needs to be digitized at the signal Nyquist rate of several gigahertz, so that all of the receiver functions are performed digitally.

In this paper, we propose a channelized digital receiver that efficiently samples the UWB signal at a fraction of the chip frequency. The proposed receiver achieves high reception performance in the presence of strong narrowband interferers and

\footnotetext{
${ }^{1}$ Although ADCs with higher resolution and speed can be designed in nonmainstream process technologies, CMOS is the least expensive. Furthermore, by integrating the CMOS ADC as part of a system-on-chip, the overall radio cost becomes significantly lower.
} 
enjoys numerous implementation advantages as described in subsequent sections.

The proposed receiver channelizes the received UWB signal using a bank of continuous-time analog analysis filters then estimates the transmitted signal using a bank of discrete-time digital synthesis filters. Although such hybrid filter banks (HFB) have been previously studied [7]-[9], they differ from the proposed receiver primarily in their objective. Unlike existing HFBs that attempt to perfectly reconstruct the received signal, the objective of the proposed receiver is to optimally estimate the transmitted signal for data detection. The synthesis filters, therefore, are designed to perform minimum mean-squared error (MMSE) estimates of the transmitted signal in the presence of additive noise, narrowband interference, and aliasing from sampling. A novel approach for designing such synthesis filters and for quantifying the resulting receiver performance is presented. The proposed receiver also differs from existing HFBs in the channelization method. Instead of using high frequency bandpass filters, which are generally difficult to realize in ICs, the proposed receiver uses mixers and simple low-pass filters to relax the implementation requirements. This paper focuses on maximally decimated HFBs, although the design techniques presented are general and can be applied to oversampled HFBs at the expense of higher sampling frequency.

This paper is organized as follows. In Section II, the proposed receiver is described. A system model of the proposed receiver is presented in Section III. In Section IV, the design and performance analysis of the synthesis filters are described. The performance results are presented in Section V. Finally, conclusions are drawn in Section VI.

\section{PROPOSED RECEIVER}

\section{A. Existing Channelization Approaches}

To sample at a fraction of the effective sampling frequency, the received analog signal needs to be channelized either in the time or frequency domain. An approach that has been used in high-speed digital sampling oscilloscopes is to employ an array of $M$ ADCs each triggered successively at $1 / M$ the effective sample rate of the parallel ADC [10], [11]. A fundamental problem with an actual implementation of such time-interleaved architecture is that each ADC sees the full bandwidth of the input signal. This causes great difficulty in the design of the sample/hold circuitry. Furthermore, in the presence of strong narrowband interferers, each ADC requires an impractically large dynamic range to resolve the signal from the narrowband interferers.

Instead of channelizing by time-interleaving, the received signal can be channelized into multiple frequency subbands with an ADC in each subband channel operating at a fraction of the effective sampling frequency [7]-[9]. A filter bank view of the frequency channelized ADC is shown in Fig. 2. A bandlimited analog input signal $g(t)$ is split into $M$ subband signals using $M$ analysis filters $\left\{H_{k}(j \Omega), 0 \leq k \leq M-1\right\}$. The resulting signals are sampled at $f_{\text {eff }} / M$, where $f_{\text {eff }}$ is the effective sampling frequency, and digitized using $M$ ADCs. Signal reconstruction is achieved by upsampling (expanding) the digitized samples by a factor of $M$, passing through the discrete synthesis filters $\left\{F_{r}(z), 0 \leq r \leq M-1\right\}$, and then summing.

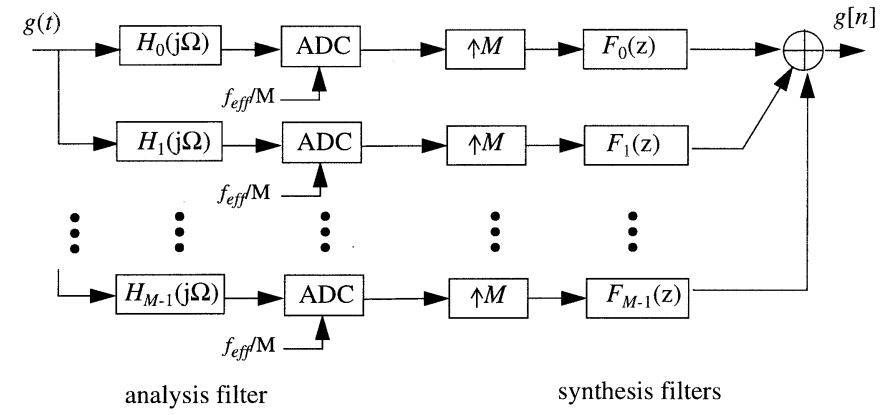

Fig. 2. $M$-channel subband ADC.

An important advantage of channelizing in the frequency domain is that the dynamic range requirement of each ADC is greatly relaxed. This advantage arises because the frequency channelization process distributes the input signal energy to each ADC and isolates the effects of large narrowband interferers. The sample/hold circuitry, however, is still very difficult to design as it sees the uppermost frequency in the high subband channels. In addition, sharp bandpass filters with high center frequencies, which are necessary to mitigate the effects of strong narrowband interferers, are extremely difficult to realize, especially in ICs.

\section{B. Proposed Channelization}

Instead of using bandpass filters with high center frequencies, channelization can be achieved using a bank of $M$ mixers operating at equally spaced frequencies and $M$ low-pass filters to decompose the analog input signal into $M$ subbands. In addition to obviating the need to design high frequency bandpass filters, channelizing the received signal using this approach greatly relaxes the design requirements of the sample/hold circuitry. The sample/hold circuitry in this architecture sees only the bandwidth of the subband signal; whereas in the bandpass channelization approach, the sample/hold circuitry sees the uppermost frequency in the high subband channels. Consequently, the required sampling aperture, which is the amount of time required for the sampler to capture the input value, is much more relaxed in the proposed channelization approach.

The analog section of the proposed receiver is shown in Fig. 3. It employs a bank of complex mixers operating at equally spaced frequencies [denoted as $f_{1}, f_{2}, \ldots, f_{M-1}$ ] and low-pass filters [denoted as $H(j \Omega)$ ] to decompose the analog input signal into $M$ subbands. The low-pass filter $H(j \Omega)$ should be designed to have sharp rolloffs with large attenuation in the stopband frequency, since it results in greater robustness to strong narrowband interferers as described in subsequent sections. The phases of the $M-1$ mixers do not affect the receiver performance as long as they are known (e.g., through training) and accounted for when designing the synthesis filters. Thus, the mixer phases are assumed to be zero for simplicity. The mixer frequencies are chosen to be multiples of each other (i.e., $f_{a}=a f_{1}, a \in\{1,2, \ldots, M-1\}$ ), because a simple frequency divider can then be used to generate the multiple frequencies. When the channelizer is maximally decimated to minimize the ADC sampling frequency, the cutoff frequency of $H(j \Omega)$ is $f_{\text {sample }} / 2$, where $f_{\text {sample }}$ is the ADC sampling frequency, and $f_{\text {sample }}=f_{1}$. Given these frequency choices, the 


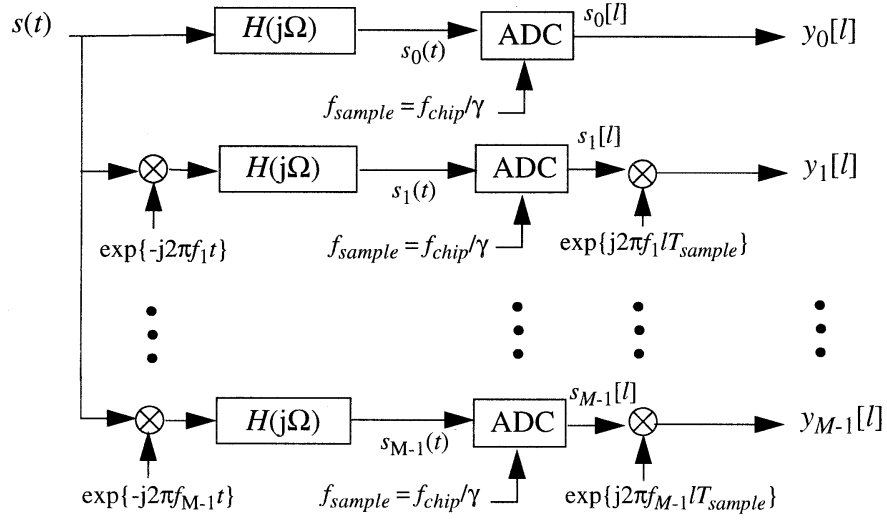

Fig. 3. Proposed channelizer with $M$ subband channels.

proposed channelizer achieves an effective sampling frequency of $(2 M-1) f_{\text {sample. }}$.

The continuous-time analysis filters in existing HFBs are generally designed to approximate the transfer functions of known perfect (or near perfect) reconstruction discrete-time analysis filters [7]-[9]. Since such approximation typically yields analysis filters that are very complex, the proposed receiver makes no such approximation. In addition, the objective of the proposed receiver is not to perfectly reconstruct the received signal but to optimally estimate the transmitted signal for data detection. Hence, there is no need to employ perfect reconstruction analysis filters, and $H(j \Omega)$ is assumed to be a simple Butterworth filter. As shown in subsequent sections, near optimal reception performance can be achieved using such simple analysis filters.

In Fig. 3, the zeroth subband signal [denoted as $s_{0}(t)$ ] is a real signal as no downconversion is performed, and the remaining subband signals [denoted as $s_{1}(t), s_{2}(t), \ldots, s_{M-1}(t)$ ] are complex signals. Thus, to achieve the effective sampling frequency of $f_{\text {eff }}$ when the proposed channelizer is maximally decimated, a total of $2 M-1$ ADCs (two ADCs per nonzero subband channel) each operating at $f_{\text {eff }} /(2 M-1)$ is required. By comparison, the bandpass channelization approach requires $M$ ADCs sampling at $f_{\text {eff }} / M$, which represents nearly half as many ADCs operating at almost twice the sampling frequency of the proposed channelizer.

Throughout this paper, we assume that $M$ and $f_{\text {eff }}$ are selected so that sampling in each ADC occurs at an integer fraction of the chip frequency, i.e., $f_{\text {sample }}=f_{\text {chip }} / \gamma$, where $f_{\text {chip }}$ is the chip frequency and $\gamma$ is an integer value. This assumption is employed to simplify the subsequent performance analysis. The sampled signals in each subband channel are then digitally modulated as shown in Fig. 3. The purpose of the digital modulator is described in the following section. The resulting $l$ th discrete signal in the $k$ th subband channel is denoted as $y_{k}[l]$.

The mixer circuit nonidealities can potentially distort the downconverted subband signals. With good circuit techniques, however, much of this distortion can be minimized. In this paper, the mixers are assumed to behave ideally.

\section{Transmitted Signal Estimation}

Instead of first reconstructing the received signal, the proposed receiver operates directly on the sampled signals

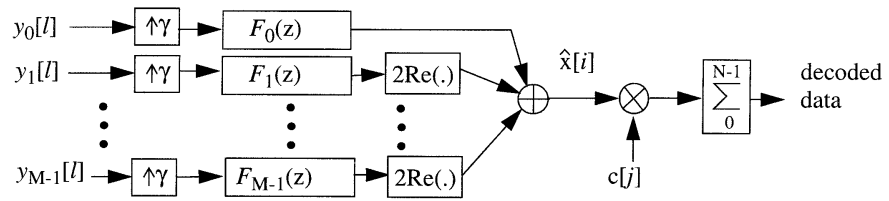

Fig. 4. Digital section of the proposed receiver.

to estimate the transmitted signal. As shown in Fig. 4, this is achieved by upsampling the digitally modulated signals $\left\{y_{k}[l], k=0,1, \ldots, M-1\right\}$ by $\gamma$ [since the sampling frequency is $(1 / \gamma)$ th the chip frequency], filtering the result by a bank of synthesis filters $\left\{F_{k}(z), k=0,1, \ldots, M-1\right\}$, converting the nonzero subband channel signals to real signals, and then summing. The conversion of the nonzero subband signals to real signals is performed since the transmitted signal is a real signal. The resulting sum value is denoted as $\hat{x}[i]$, which is the estimate of the $i$ th transmitted signal $x[i]$. This estimate is multiplied by the corresponding pseudorandom spreading code, $c[j]$, then summed for the duration of the spreading factor $N$ as shown in Fig. 4.

The synthesis filters are designed to perform MMSE estimates of the transmitted signal in the presence of multipaths, narrowband interferers, additive noise, and aliasing from sampling. To design such filters, the proposed receiver is viewed as $\gamma$ parallel receivers that linearly estimate blocks of $\gamma$ consecutive transmitted signals. As described in Section IV, this perspective allows the use of known linear estimation techniques to design synthesis filters that optimally estimate the transmitted signals in the MMSE sense. In addition, this view enables the use of existing adaptive algorithms [12] to appropriately adjust the synthesis filter taps to variations in the additive noise, the analog filters, and the propagation channel. The adaptive performance of the proposed receiver, however, is not addressed in this paper.

\section{SYSTEM MODEL}

In this paper, parenthesis and brackets are used to distinguish between continuous-time and discrete-time signals, respectively. Bold characters are used to denote vectors or matrices.

\section{A. Received Signal Model}

The overall system model is shown in Fig. 5. The $m$ th incoming bit stream $b[m]$, where $b[m] \in\{1,-1\}$, is spread by the $j$ th pseudorandom (PN) code $c[j]$, where $c[j] \in\{1,-1\}$, to obtain the $i$ th transmitted signal $x[i]$, where $i=j+m N$. The period of the PN code is $N$, which is also the spreading factor. Signal $x[i]$ is scaled by $\|p\|$, then passed at a rate of $f_{\text {chip }}$ through a normalized transmit filter $\varphi_{\operatorname{tr}}(t)$ to obtain $x(t)$. The resulting signal power is $\|p\|^{2}$. Signal $x(t)$ is then filtered by the transmit antenna, the propagation channel, and the receive antenna, whose impulse responses are denoted as $a_{\mathrm{tr}}(t), u(t)$, and $a_{r}(t)$, respectively. The resulting signal is corrupted by $n(t)$, which consists of an additive white Gaussian noise (AWGN) of two-sided noise power spectral density equal to $N_{0} / 2$ and a narrowband interferer $I(t)$. The corrupted signal is then passed through an anti-alias filter $\varphi_{\text {alias }}(t)$, which is assumed to be an ideal low-pass filter with a gain transfer of $\sqrt{1 / f_{\text {eff }}}$ over the 


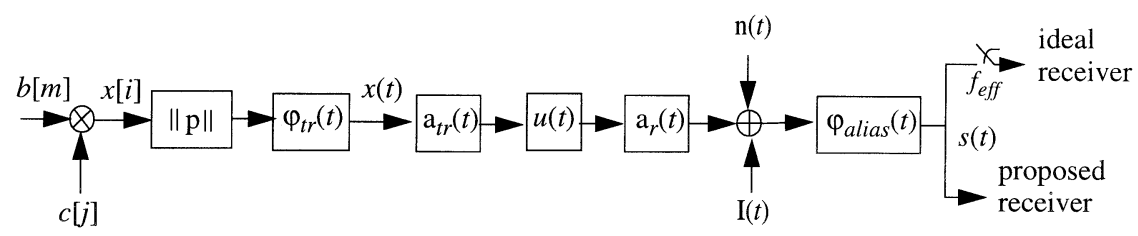

Fig. 5. Overall system model.

frequency range of $-\pi f_{\text {eff }} \leq \Omega \leq \pi f_{\text {eff }}$. For comparison purposes, the resulting signal $s(t)$ is the input to both the proposed receiver and the ideal conventional receiver, which is defined as a receiver that samples at $f_{\text {eff }}$, then employs an infinite length linear filter to estimate the transmitted signal $x[$.$] before cor-$ relating. Although the anti-alias filter is not needed in the proposed receiver, it is employed so that a fair comparison can be made between the two receivers.

The transmit filter $\varphi_{\operatorname{tr}}(t)$ is modeled as a Gaussian pulse with a standard deviation of $\sigma$. The Gaussian model is employed so that the pulse is differentiable when passing through the antennas, which are modeled as differentiators [16]

$$
a_{\mathrm{tr}}(t)=a_{r}(t)=\frac{d}{d t}(.) \text {. }
$$

The received signal at the output of the anti-alias filter is given by

$$
\begin{aligned}
s(t) & =\sum_{m} \sum_{j=0}^{N-1}\|p\| b[m] c[j] \varphi_{p}\left(t-j T_{\text {chip }}-m N T_{\text {chip }}\right)+n_{p}(t) \\
& =\sum_{i}\|p\| x[i] \varphi_{p}\left(t-i T_{\text {chip }}\right)+n_{p}(t)
\end{aligned}
$$

where $i=j+m N$

$$
\begin{aligned}
& \varphi_{p}(t)=\varphi_{\mathrm{tr}}^{\prime \prime}(t) \otimes u(t) \otimes \varphi_{\text {alias }}(t) \\
& n_{p}(t)=(n(t)+I(t)) \otimes \varphi_{\text {alias }}(t) .
\end{aligned}
$$

$\otimes \quad$ denotes convolution;

$b[m] \quad m$ th binary data bit;

$c[j] \quad j$ th code in a PN sequence of period $N$;

$x[i] \quad b[m] c[j] ; i$ th transmitted signal;

$\|p\| \quad$ amplitude of the transmitted signal;

$T_{\text {chip }} \quad$ code chip period (i.e., $1 / f_{\text {chip }}$ );

$\varphi_{\mathrm{tr}}^{\prime \prime}(t)$ second derivative of normalized transmit filter $\varphi_{\mathrm{tr}}(t)$;

$u(t) \quad$ propagation channel response;

$n_{p}(t)$ additive white Gaussian noise with two-sided spectral density of $N_{0} / 2$;

$I(t) \quad$ narrowband interferer; assumed to be colored Gaussian noise.

\section{B. Discrete Channel Model of the Proposed Channelizer}

The signal in the $k$ th subband channel just before the sampling device (see Fig. 3) can be written as

$$
s_{k}(t)=\sum_{i} x[i] e^{-j \Omega_{k} t} p_{k}\left(t-i T_{\text {chip }}\right)+n_{p}(t) e^{-j \Omega_{k} t} \otimes h(t)
$$

where $\Omega_{k}=2 \pi f_{k}$ with $f_{0}=0, h(t)$ is the impulse response of $H(j \Omega)$, and

$$
p_{k}(t)=\|p\| \varphi_{p}(t) \otimes e^{j \Omega_{k} t} h(t) .
$$

Sampling at time instant $t=l T_{\text {sample }}=\gamma l T_{\text {chip }}$, where $T_{\text {sample }}$ is the ADC sampling period, the resulting signal after quantization is

$$
s_{k}[l]=s_{k}\left(\gamma l T_{\text {chip }}\right)+n_{q_{k}}[l]
$$

where $n_{q_{k}}[l]$ is the quantization noise of the $l$ th sample in the $k$ th subband channel.

We assume that the quantization noise is a random noise whose variance is proportional to the variance of the input to the ADC. Such a constraint accurately describes the interaction between the quantization noise and the dynamic range of the ADC. Assuming $s_{k}\left(\gamma l T_{\text {chip }}\right)$ is approximately Gaussian distributed with a standard deviation of $\sigma_{s_{k}}$ and the allowed input range to the ADC before overflowing is $3 \sigma_{s_{k}}$, the variance of the quantization noise in the $k$ th subband channel (denoted as $\left.\sigma_{q_{k}}^{2}\right)$ is

$$
\sigma_{q_{k}}^{2}=0.75 \cdot 2^{-2(b-1)} \cdot \sigma_{s_{k}}^{2}
$$

where $b$ is the number of ADC bits [17]. The Gaussian approximation is reasonably accurate since $n_{p}(t)$, which typically dominates the total received signal power in an UWB system, is approximately Gaussian distributed. We assume throughout this paper that the variable gain amplifier (VGA) in each subband channel appropriately scales the channelized signal such that the quantization noise is accurately described by (8).

The quantized sample $s_{k}[l]$ given in (7) is in general timevarying. This time variation, which can complicate our subsequent analysis, can be eliminated by digitally modulating $s_{k}[l]$ by $e^{j \Omega_{k} \gamma l T_{\text {chip }}}$ as in Fig. 3. The resulting signal $y_{k}[l]$ then becomes time-invariant. When the proposed channelizer is maximally decimated (i.e., $f_{\text {sample }}=f_{1}$ ), however, the digital modulators become unnecessary, since $e^{j \Omega_{k} \gamma l T_{\text {chip }}}=1$ for all $l$ and $k$.

Another potential mathematical difficulty arises when converting the output of the nonzero subband synthesis filters to a real signal as shown in Fig. 4. This difficulty, however, can be avoided by assuming for analysis purposes only that each nonzero subband channel generates both the signal and its conjugate. The conjugate signal is then filtered by the corresponding conjugate synthesis filter [e.g., $F_{k}^{*}(z)$ ] as shown in Fig. 6. The operations performed in Fig. 6, which are equivalent in effect to the operations performed in Fig. 4, are mathematically convenient and subsequently employed in our analysis. For notational simplicity, the signal and its conjugate in the nonzero subband channels are grouped and represented as a two-element vector, e.g., $\boldsymbol{y}_{k}[l]=\left[y_{k}[l] y_{k}^{*}[l]\right]^{T}$.

After digitally modulating, the resulting signals for all $M$ subband channels can be represented using vectors as

$$
\boldsymbol{y}[l]=\sum_{i} x[i] \cdot \boldsymbol{p}[\gamma l-i]+\boldsymbol{n}[l]
$$




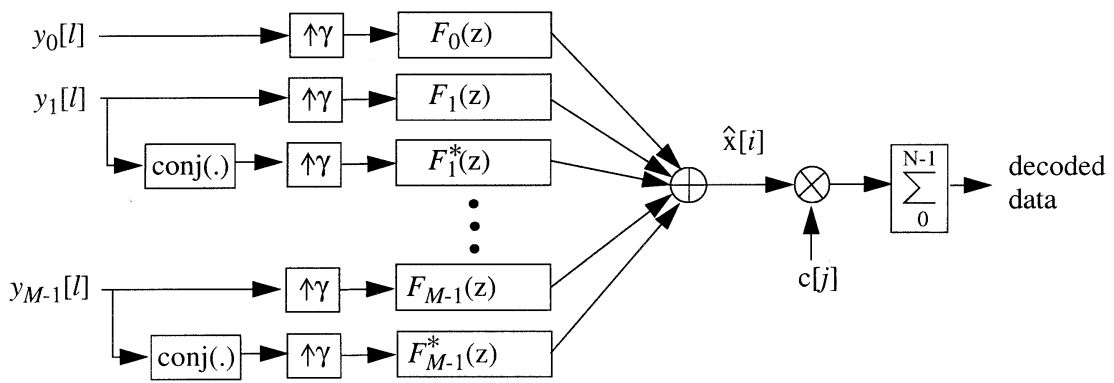

Fig. 6. Equivalent digital section of proposed receiver.

where

$$
\begin{gathered}
\boldsymbol{y}[l] \triangleq\left[\begin{array}{c}
y_{0}[l] \\
\boldsymbol{y}_{1}[l] \\
\vdots \\
\boldsymbol{y}_{M-1}[l]
\end{array}\right]=\left[\begin{array}{c}
s_{0}[l] \\
s_{1}[l] e^{j \Omega_{1} \gamma l T_{\text {chip }}} \\
s_{1}^{*}[l] e^{-j \Omega_{1} \gamma l T_{\text {chip }}} \\
\vdots \\
s_{M-1}[l] e^{j \Omega_{M-1} \gamma l T_{\text {chip }}} \\
s_{M-1}^{*}[l] e^{-j \Omega_{M-1} \gamma l T_{\text {chip }}}
\end{array}\right] \\
\boldsymbol{p}[l] \triangleq\left[\begin{array}{c}
p_{0}[l] \\
\boldsymbol{p}_{1}[l] \\
\vdots \\
p_{M-1}\left(l T_{\text {chip }}\right) \\
p_{1}\left(l T_{\text {chip }}\right) \\
p_{1}^{*}\left(l T_{\text {chip }}\right) \\
\vdots \\
p_{M-1}\left(l T_{\text {chip }}\right) \\
p_{M-1}^{*}\left(l T_{\text {chip }}\right)
\end{array}\right]
\end{gathered}
$$

and

$$
\begin{aligned}
& \boldsymbol{n}[l] \triangleq\left[\begin{array}{c}
n_{0}[l] \\
\boldsymbol{n}_{1}[l] \\
\vdots \\
\boldsymbol{n}_{M-1}[l]
\end{array}\right]
\end{aligned}
$$

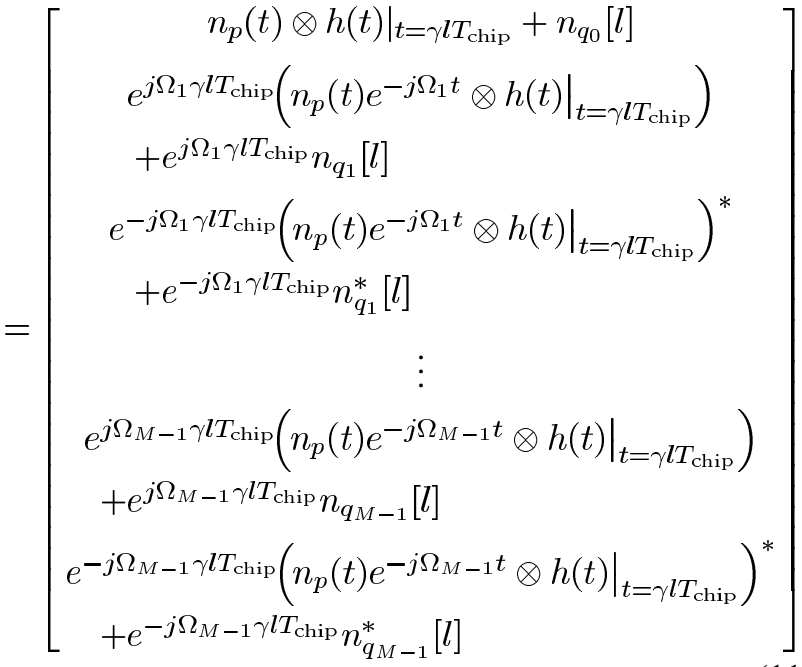

Since both the signal and its conjugate are needed in all nonzero subband channels, vector $\boldsymbol{y}[l]$ consists of $2 M-1$ elements.

The sampled pulse response $\boldsymbol{p}[l]$ is assumed to extend only a finite time interval, and any nonzero component of $p_{k}(t)$ outside

and

$$
\begin{aligned}
R_{n_{m} n_{q}}[\alpha]= & \frac{1}{2 \pi} \int_{-\pi f_{\text {eff }}}^{\pi f_{\text {eff }}} S_{n_{p}}(j \Omega) H\left(j\left(\Omega-\Omega_{m}\right)\right) \\
& \cdot H^{*}\left(j\left(\Omega-\Omega_{q}\right)\right) e^{j \Omega \alpha T_{\text {sample }}} d \Omega \\
& +\sigma_{q_{m}}^{2} \cdot \delta[\alpha] \cdot \delta[m-q]
\end{aligned}
$$

The discrete channel model with $M$ subband channels is shown in Fig. 7. The noise correlation function is readily computed as

$$
E\left\{\boldsymbol{n}_{m}[l+\alpha] \boldsymbol{n}_{q}^{H}[l]\right\}=\left[\begin{array}{ll}
R_{n_{m} n_{q}}[\alpha] & R_{n_{m} n_{q}^{*}}[\alpha] \\
R_{n_{m} n_{q}^{*}}^{*}[\alpha] & R_{n_{m} n_{q}}[\alpha]
\end{array}\right]
$$

where

$$
\begin{aligned}
R_{n_{m} n_{q}^{*}}[\alpha]= & \frac{1}{2 \pi} \int_{-\pi f_{\text {eff }}}^{\pi f_{\text {eff }}} S_{n_{p}}(j \Omega) H\left(j\left(\Omega-\Omega_{m}\right)\right) \\
& \cdot H\left(-j\left(\Omega-\Omega_{q}\right)\right) e^{j \Omega \alpha T_{\text {sample }}} d \Omega .
\end{aligned}
$$


$S_{n_{p}}(j \Omega)$ is the power spectral density of $n_{p}(t)$ and $\delta[$.$] is the$ Dirac unit pulse. Note that the digital modulator in each subband channel not only makes the channel response time-invariant, but the noise correlation functions become wide-sense stationary (WSS).

\section{Synthesis Filter DESIGN AND PERFORMANCE ANALysis}

The synthesis filter bank shown in Fig. 4 performs matched filtering, noise whitening, channel equalization, and aliasing cancellation. To design such synthesis filters, the synthesis filter bank, which is a linear periodically time varying (LPTV) system with period $\gamma$, is reformulated as $\gamma$ parallel receivers that linearly estimate blocks of $\gamma$ consecutive transmitted signals. The estimates of these $\gamma$ parallel receivers at the $l$ th time instant, denoted as $\left\{\hat{x}_{k}[l], k=0,1, \ldots, \gamma-1\right\}$, are MMSE estimates of transmitted signals $\{x[\gamma l-\Delta-k], k=0,1, \ldots, \gamma-1\}$, where $\Delta$ is the system delay.

The estimate $\hat{x}_{k}[l](=\hat{x}[\gamma l-\Delta-k])$ can be expressed as the output of a linear filter, $\boldsymbol{W}_{k}$, operating on $N_{f}$ successive samples $\boldsymbol{Y}_{N_{f}}[l]$, where $\gamma N_{f}$ is the length of each synthesis filter:

$$
\hat{x}_{k}[l]=\boldsymbol{W}_{k}^{T} \boldsymbol{Y}_{N_{f}}[l]
$$

$\boldsymbol{W}_{k}$ is obtained by interleaving the synthesis filter taps by

$$
\begin{array}{r}
\boldsymbol{W}_{k} \triangleq\left[f_{0}[k] f_{1}[k] f_{1}^{*}[k] \cdots f_{M-1}[k] f_{M-1}^{*}[k] f_{0}[k+\gamma] \cdots\right. \\
\left.f_{M-1}^{*}\left[k+\gamma\left(N_{f}-1\right)\right]\right]^{T}
\end{array}
$$

where $f_{r}[m]$ is the $m$ th tap of synthesis filter $F_{r}(z)$ and $Y_{N_{f}}[l]$ is as shown in (18)-(20), at the bottom of the page. In (20), $\boldsymbol{P}_{N_{f}}$ denotes the pulse matrix of dimension $N_{f}(2 M-1) \times\left(\gamma\left(N_{f}-\right.\right.$ $1)+\nu+1), \boldsymbol{X}_{N_{f}}[l]$ the data vector of dimension $\left(\gamma\left(N_{f}-\right.\right.$
1) $+\nu+1) \times 1, N_{N_{f}}[l]$ and the noise vector of dimension $N_{f}(2 M-1) \times 1$.

Since $\left\{F_{r}(z), 0 \leq r \leq M-1\right\}$ is completely specified by $\left\{W_{k}, k=0,1, \ldots \gamma-1\right\}$, the problem of designing synthesis filters is reduced to designing $\gamma$ linear filters $\left\{\boldsymbol{W}_{k}, k=\right.$ $0,1, \ldots \gamma-1\}$ that estimate the transmitted signals $\{x[\gamma l-$ $\Delta-k], k=0,1, \ldots \gamma-1\}$. In addition to simplifying the synthesis filter design problem, an important practical advantage of this perspective is that the receiver can be made adaptive, since adaptive filters are often implemented with linear structures. The adaptive performance of the proposed receiver, however, is not discussed further in this paper.

Using the orthogonality principle, $W_{k}$ that minimizes the MSE estimate of the transmitted signal is

$$
\boldsymbol{W}_{k}=R_{x \boldsymbol{Y}_{N_{f}}} R_{\boldsymbol{Y}_{N_{f}} \boldsymbol{Y}_{N_{f}}}^{-1}
$$

where

$$
\begin{aligned}
R_{x \boldsymbol{Y}_{N_{f}}} & \triangleq E\left\{x[\gamma l-\Delta-k] \boldsymbol{Y}_{N_{f}}^{H}[l]\right\} \\
& =E\left\{x[\gamma l-\Delta-k] \boldsymbol{X}_{N_{f}}^{H}[l]\right\} P_{N_{f}}^{H}
\end{aligned}
$$

and

$$
\begin{aligned}
R_{\boldsymbol{Y}_{N_{f}} \boldsymbol{Y}_{N_{f}}} & \triangleq E\left\{\boldsymbol{Y}_{N_{f}}[l] \boldsymbol{Y}_{N_{f}}^{H}[l]\right\} \\
& =\boldsymbol{P}_{N_{f}} \cdot E\left\{\boldsymbol{X}_{N_{f}}[l] \boldsymbol{X}_{N_{f}}^{H}[l]\right\} \cdot \boldsymbol{P}_{N_{f}}^{H}+E\left\{\boldsymbol{N}_{N_{f}}[l] \boldsymbol{N}_{N_{f}}^{H}[l]\right\} \\
& \triangleq \boldsymbol{P}_{N_{f}} \cdot R_{\boldsymbol{X}_{N_{f}} \boldsymbol{X}_{N_{f}}} \cdot \boldsymbol{P}_{N_{f}}^{H}+R_{\boldsymbol{N}_{N_{f}} \boldsymbol{N}_{N_{f}}} \cdot
\end{aligned}
$$

$E\left\{x[\gamma l-\Delta-k] \boldsymbol{X}_{N_{f}}^{H}[l]\right\}$ in (22) is a $1 \times\left(\gamma\left(N_{f}-1\right)+\nu+1\right)$ vector of zeros and a one in the $(\Delta+k)$ th position, since the transmitted signal $x[$.$] is a data-modulated PN code and assumed$

$$
\begin{aligned}
& \boldsymbol{Y}_{N_{f}}[l] \triangleq\left[\begin{array}{c}
\boldsymbol{y}[l] \\
\boldsymbol{y}[l-1] \\
\vdots \\
\boldsymbol{y}\left[l-N_{f}+1\right]
\end{array}\right] \\
& =\left[\begin{array}{ccccccccccc}
\boldsymbol{p}[0] & \boldsymbol{p}[1] & \cdots & \boldsymbol{p}[\gamma] & \boldsymbol{p}[\gamma+1] & \cdots & \boldsymbol{p}[\nu] & 0 & \cdots & \cdots & 0 \\
0 & \cdots & 0 & \boldsymbol{p}[0] & \boldsymbol{p}[1] & \cdots & \cdots & \cdots & \boldsymbol{p}[\nu] & \cdots & 0 \\
\vdots & \vdots & \vdots & \vdots & \vdots & \vdots & \vdots & \vdots & \vdots & \vdots & \vdots \\
0 & \cdots & \cdots & \cdots & \cdots & 0 & 0 & \boldsymbol{p}[0] & \boldsymbol{p}[1] & \cdots & \boldsymbol{p}[\nu]
\end{array}\right]\left[\begin{array}{c}
x[\gamma l] \\
x[\gamma l-1] \\
\vdots \\
\vdots \\
x\left[\gamma\left(l-N_{f}+1\right)-\nu\right]
\end{array}\right] \\
& +\left[\begin{array}{c}
\boldsymbol{n}[l] \\
\boldsymbol{n}[l-1] \\
\vdots \\
\boldsymbol{n}\left[l-N_{f}+1\right]
\end{array}\right] \\
& \triangleq \boldsymbol{P}_{N_{f}} \cdot \boldsymbol{X}_{N_{f}}[l]+\boldsymbol{N}_{N_{f}}[l]
\end{aligned}
$$


to be white. $R_{\boldsymbol{X}_{N_{f}} \boldsymbol{X}_{N_{f}}}$ in (23) is an identity matrix for the same reason, and $R_{\boldsymbol{N}_{N_{f}} \boldsymbol{N}_{N_{f}}}$ is readily obtained using (13).

The unbiased signal-to-noise ratio (SNR) of the transmitted signal estimate $\hat{x}_{k}[l]$, which is defined as $E\left\{x^{2}[\gamma l-\Delta-k]\right\} / E\left\{\left(\hat{x}_{k}[l]-x[\gamma l-\Delta-k]\right)^{2}\right\}$ with $E\left\{\hat{x}_{k}[l] \mid x[\gamma l-\Delta-k]\right\}=x[\gamma l-\Delta-k]$, is [19]

$$
\mathrm{SNR}_{k}=\frac{\boldsymbol{W}_{k} R_{x \boldsymbol{Y}_{N_{f}}}^{H}}{1-\boldsymbol{W}_{k} R_{x \boldsymbol{Y}_{N_{f}}}^{H}} .
$$

Since the synthesis filter bank is a LPTV system with period $\gamma$, the unbiased spread SNR of the proposed receiver is the average unbiased SNR of $\gamma$ consecutive transmitted signal estimates, i.e.,

$$
\mathrm{SNR}_{\text {proposed }}=\frac{1}{\gamma} \cdot \sum_{i=0}^{\gamma-1} \mathrm{SNR}_{i} .
$$

The despread (or symbol) SNR can be readily obtained by multiplying (25) by the spreading factor $N$. To achieve the highest reception performance, $\Delta$ should be selected so that $\mathrm{SNR}_{\text {proposed }}$ is maximized.

\section{Computational Results AND Discussion}

In this section, the performance of the proposed receiver given in (25) is compared against that of an ideal conventional receiver. As defined earlier, an ideal conventional receiver is a receiver that samples at $f_{\text {eff }}$ then employs an infinite length linear filter to estimate the transmitted signal before correlating. The unbiased spread SNR of an ideal conventional receiver is denoted as $\mathrm{SNR}_{\text {ideal }}$. As a reference for comparison, the matched filter bound (MFB) when no narrowband interferer is present is computed. This bound is subsequently referred to as $\mathrm{SNR}_{\text {bound }}$ and is given by

$$
\mathrm{SNR}_{\text {bound }}=\frac{\|p\|^{2}}{N_{0} / 2} .
$$

Throughout this section, the following assumptions are made.

1) The channelizer is maximally decimated.

2) The sampling frequency $f_{\text {sample }}$ is $f_{\text {chip }} / \gamma$, where $\gamma \in$ $\{1,2,3\}$.

3) The mixer frequency $f_{i}$ is $i \cdot f_{\text {sample, where }}$ $i \in\{0,1,2, \ldots, M-1\}$.

4) $H(j \Omega)$ is a Butterworth filter with cutoff frequency of $f_{\text {sample }} / 2$. It is a fourth-order filter unless specified otherwise.

5) The propagation channel response $u(t)$ is a delta function.

6) The chip frequency $f_{\text {chip }}$ is $0.35 / \sigma$, where $\sigma$ is the standard deviation of Gaussian transmit pulse.

7) The effective sampling frequency $f_{\text {eff }}$ is $3 f_{\text {chip }}$ unless specified otherwise.

8) $I(t)$ is a real brickwall narrowband interferer with center frequency $I_{f}$ and magnitude $I_{\text {mag }}$ greater than $N_{0} / 2$. For ease of presentation, the bandwidth of $I(t)$ is fixed at $15 \%$ of $f_{\text {chip }}$.

In Fig. 8, SNR proposed given in (25) and $\mathrm{SNR}_{\text {ideal }}$ are plotted against $N_{f}$ when no narrowband interferer is present

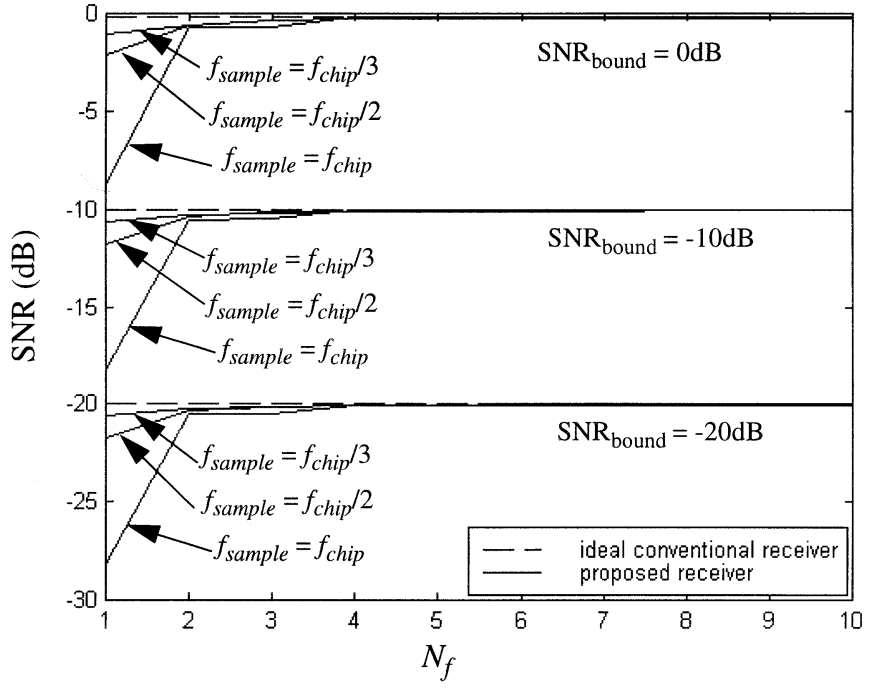

Fig. 8. SNR versus $N_{f}$ with no interferer present.

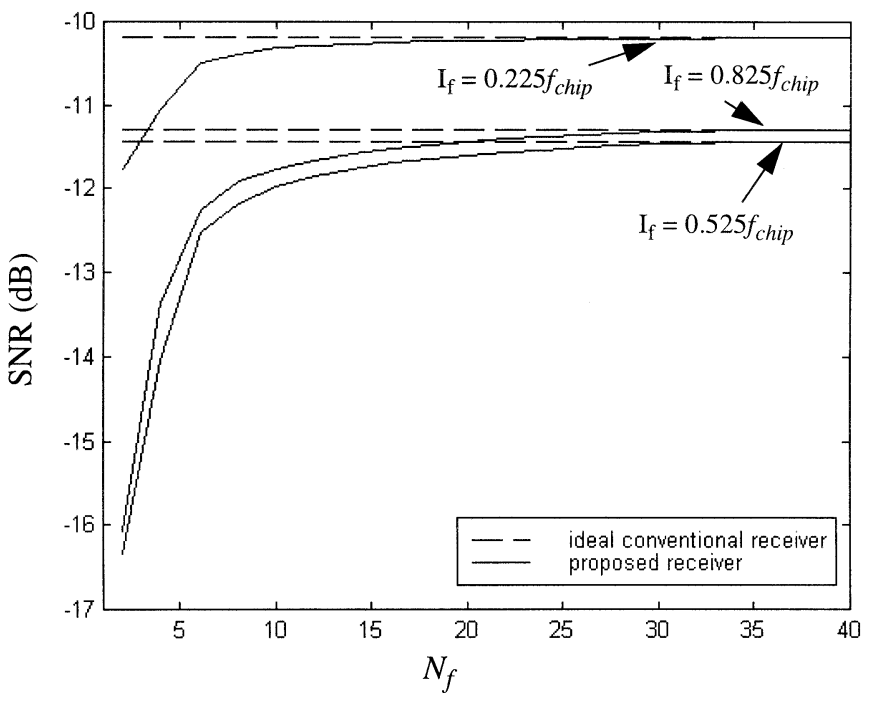

Fig. 9. SNR versus $N_{f}$ with $I_{\text {mag }}=60 \mathrm{~dB}$.

[i.e., $I(t)=0$ ]. For $\mathrm{SNR}_{\text {bound }}=0 \mathrm{~dB},-10 \mathrm{~dB},-20 \mathrm{~dB}$, the performance of the proposed receiver is plotted assuming: $\gamma=1, M=2, f_{\text {eff }}=3 f_{\text {chip }} ; \gamma=2, M=4, f_{\text {eff }}=3.5 f_{\text {chip }}$; and $\gamma=3, M=5, f_{\text {eff }}=3 f_{\text {chip. }}$. As shown in Fig. 8, the proposed receiver requires $N_{f}$ of approximately five (or synthesis filter taps of approximately $5 \gamma$ ) to achieve near optimal receiver performance.

In the presence of a large narrowband interferer, significantly more filter taps are required. Fig. 9 plots $\mathrm{SNR}_{\text {proposed }}$ and $\mathrm{SNR}_{\text {ideal }}$ against $N_{f}$ in the presence of a narrowband interferer when $M=5, \mathrm{SNR}_{\text {bound }}=-10 \mathrm{~dB}$, and $f_{\text {sample }}=f_{\text {chip }} / 3$. The center frequencies of the narrowband interferer, $I_{f}$, are $0.225 f_{\text {chip }}, 0.525 f_{\text {chip }}$, and $0.825 f_{\text {chip }}$ each with $I_{\text {mag }}=60 \mathrm{~dB}$. Near optimal receiver performance is achieved for $N_{f}$ greater than or equal to 30 , which is significantly more compared with when no narrowband interferer is present. This increase arises because the synthesis filters must also suppress the effects of the narrowband interferer.

Fig. 10 plots $\mathrm{SNR}_{\text {proposed }}$ and $\mathrm{SNR}_{\text {ideal }}$ as a function of $I_{f}$ for $I_{\text {mag }}=30 \mathrm{~dB}, 70 \mathrm{~dB}$. We assume that $\mathrm{SNR}_{\text {bound }}=$ 


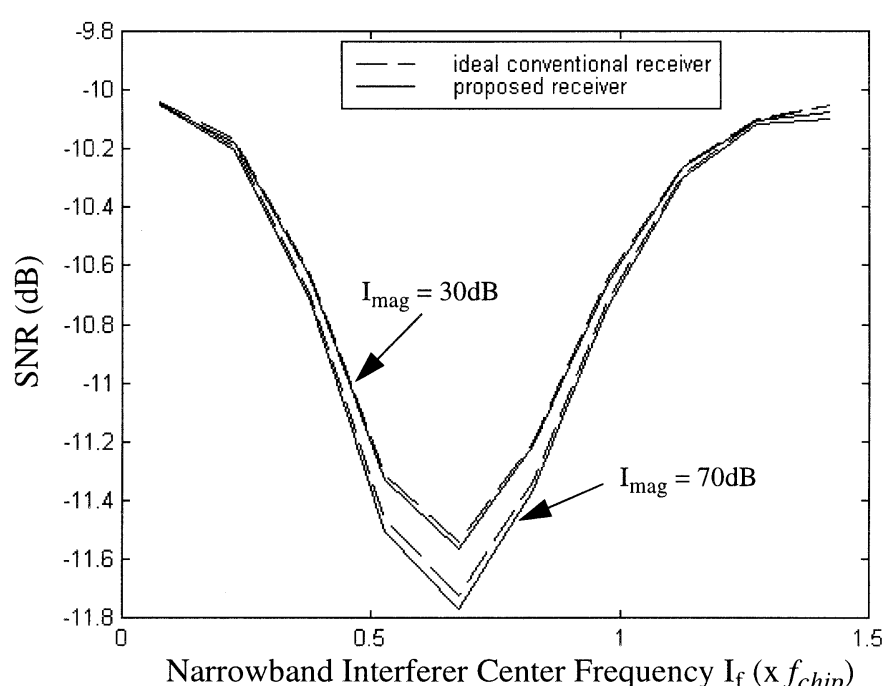

Fig. 10. SNR versus interference center frequency $\left(I_{f}\right)$.

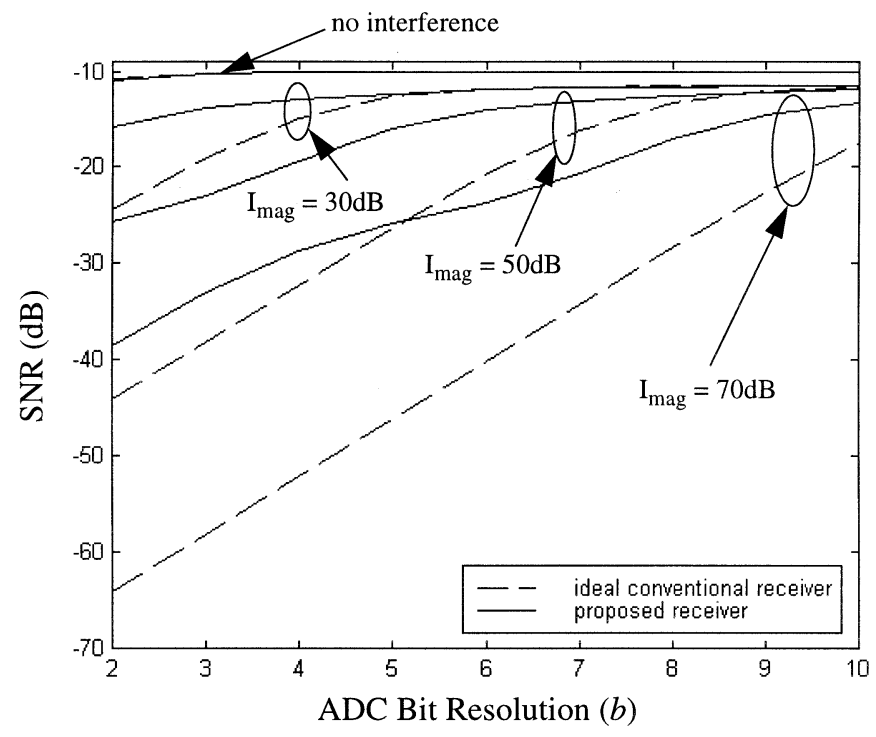

Fig. 11. SNR versus ADC bits $(b)$ assuming $H(j \Omega)$ is a fourth-order Butterworth filter.

$-10 \mathrm{~dB}, M=5, f_{\text {sample }}=f_{\text {chip }} / 3$, and $N_{f}=30$. In Fig. 10 , the proposed receiver achieves the worst performance when $I_{f}$ is approximately $0.675 f_{\text {chip }}$. This frequency value corresponds to the region where the signal spectral density is the highest. For the remaining results, we only consider the worst interferer and assume that $I_{f}=0.675 f_{\text {chip }}$.

In Fig. 11, $\mathrm{SNR}_{\text {proposed }}$ and $\mathrm{SNR}_{\text {ideal }}$ are plotted against the number of ADC bits. The number of bits, $b$, allocated to each $\mathrm{ADC}$ in the proposed architecture is the same for all subband channels. For comparison purposes, the ideal conventional receiver also employs $b$ ADC bits. We assume in Fig. 11 that $\mathrm{SNR}_{\text {bound }}=-10 \mathrm{~dB}, M=5, f_{\text {sample }}=f_{\text {chip }} / 3$, and $N_{f}=$ 30 . When no narrowband interferer is present, there is very little difference in performance between the two receivers. However, in the presence of a narrowband interferer, the proposed receiver outperforms the ideal conventional receiver with the difference increasing as $I_{\text {mag }}$ increases and $b$ decreases. For example, the

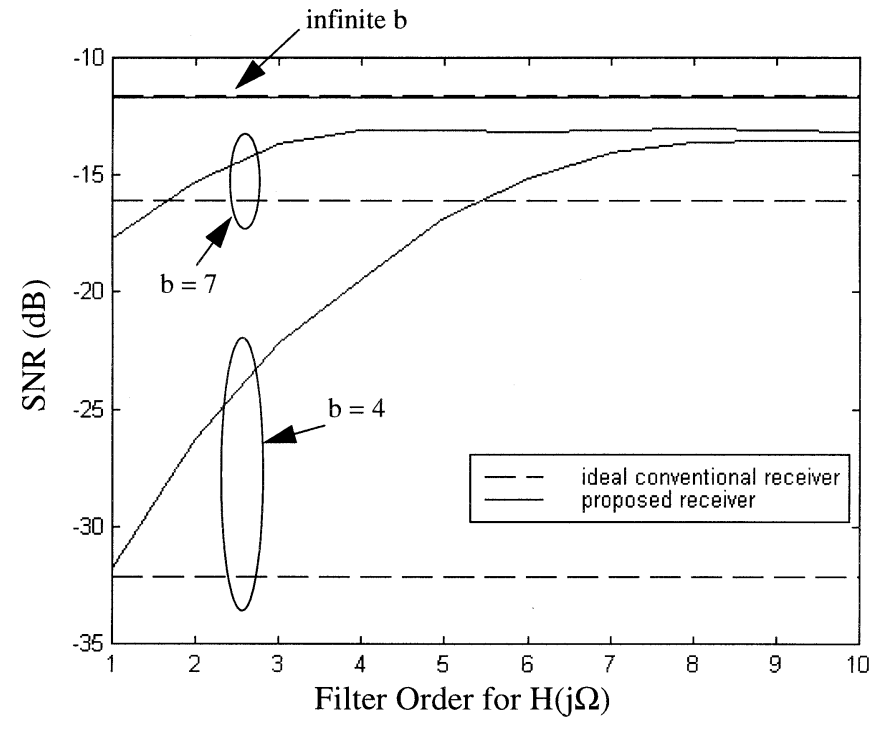

Fig. 12. SNR versus filter order for $I_{\operatorname{mag}}=50 \mathrm{~dB}$.

spread SNR in the proposed receiver is 13 and $24 \mathrm{~dB}$ higher than the ideal conventional receiver when a 4-bit ADC is employed in the presence of 50 and $70 \mathrm{~dB}$ narrowband interferers, respectively.

This large performance difference arises because the channelization process in the proposed receiver isolates the effects of the narrowband interferer by raising the quantization noise floor mostly in the subband channels containing the interferers. Since significant interference noise is already present in these subband channels, the additional quantization noise does not significantly increase the total noise power relative to the signal power. By contrast, a narrowband interferer in the conventional receiver increases the quantization noise floor across the entire signal spectrum. Thus, even in frequencies with no interference, the quantization noise floor is significantly raised relative to the signal power, resulting in large overall performance degradation.

Since frequency channelization isolates the effects of the narrowband interferer, the proposed receiver achieves greater robustness to a narrowband interferer when sharper channelization filters are employed. This effect is illustrated in Fig. 12, which plots $\mathrm{SNR}_{\text {proposed }}$ against the filter order of $H(j \Omega)$. For comparison purposes, $\mathrm{SNR}_{\text {ideal }}$ is also plotted. The assumptions in this figure are $\mathrm{SNR}_{\text {bound }}=-10 \mathrm{~dB}$, $M=5, I_{\text {mag }}=50 \mathrm{~dB}, f_{\text {sample }}=f_{\text {chip }} / 3$, and $N_{f}=30$. If infinite bit resolution is available, the filter order does not affect the receiver performance. However, when there is only a finite number of ADC bits, sharper filters better isolate the increase in the quantization noise power caused by the narrowband interferer. In Fig. 12, the performance of the proposed receiver improves steadily compared with the ideal conventional receiver as the filter order increases. This improvement saturates when the filter order is approximately four for $b=7$ and eight for $b=4$, which correspond to performance improvements of roughly 3 and $20 \mathrm{~dB}$, respectively. Thus, significant performance improvements are possible by increasing the filter order, especially when the available ADC resolution is low. 


\section{CONCLUSION}

A channelized digital UWB receiver that efficiently samples the UWB signal at a fraction of the chip frequency is presented. After sampling at a much reduced frequency, the digital synthesis filter bank performs MMSE estimates of the transmitted signals in the presence of narrowband interferers, additive noise, and aliasing from sampling. An approach for designing such synthesis filters and for quantifying the resulting receiver performance is described.

The received UWB signal is channelized into multiple frequency subbands using a bank of mixers and low-pass filters. This channelization approach relaxes the design requirements of the sample/hold circuitry and allows the use of sharp low-pass filters, which results in greater robustness to narrowband interferers. Another important implementation advantage is that the proposed receiver architecture greatly relaxes the dynamic range requirement of the ADC compared with a conventional ideal receiver. The numerous implementation advantages of the proposed UWB receiver makes it well-suited for high-performance and integrated monolithic realization.

\section{REFERENCES}

[1] M. Win and R. Scholtz, "On the robustness of ultra-wide bandwidth signals in dense multipath environments," IEEE Commun. Lett., vol. 2, pp. 51-53, Feb. 1998.

[2] T. Mitchell, "Broad is the way," IEE Review, vol. 47, no. 1, pp. 35-39, Jan. 2001.

[3] R. Scholtz, "Multiple access with time-hopping impulse modulation," in Proc. IEEE MILCOM '93, Boston, MA, Oct. 11-14, 1993, pp. 447-450.

[4] M. Win and R. Scholtz, "Impulse radio: How it works," IEEE Commun. Lett., vol. 2, pp. 36-38, Feb. 1998.

[5] R. Fleming and C. Kushner, "Low-power, miniature, distributed position location and communication devices using ultra-wideband nonsinusoidal communication technology," ARPA/FBI, Semi-Annual Tech. Rep., July 1995.

[6] M. Choi and A. A. Abidi, "A 6b 1.3 GSample/s A/D converter in $0.35 \mu \mathrm{m}$ CMOS," in Proc. IEEE Int. Solid-State Circuits Conf., vol. 438, 2001, pp. $126-127$.

[7] S. Velazquez, T. Nguyen, S. Broadstone, and J. Roberge, "A hybrid filter bank approach to analog-to-digital conversion," in Proc. IEEE-SP Int. Symp. Time-Frequency Time-Scale Anal., Oct. 1994, pp. 116-119.
[8] O. Oliaei, "Asymptotically perfect reconstruction in hybrid filter banks," in Proc. IEEE Int. Conf. Acoustics, Speech, Signal Processing, vol. 3, 1998, pp. 1829-1832.

[9] P. Loweborg, H. Johansson, and L. Wanhammar, "A class of two-channel approximately perfect reconstruction hybrid analog/digital filter banks," in Proc. IEEE Int. Symp. Circuits and Systems, vol. 1, 2000.

[10] A. Petraglia and S. Mitra, "Analysis of mismatch effects among A/D converters in a time-interleaved waveform digitizer," IEEE Trans. Instrum. Meas., vol. 40, pp. 831-835, Oct. 1991.

[11] R. Khoini-Poorard, "Mismatch effects in time-interleaved oversampling converters," in Proc. IEEE Int. Symp. Circuits Systems, London, U.K., May 1994, pp. 429-432.

[12] S. Haykin, Adaptive Filter Theory, 3rd ed. Englewood Cliffs, NJ: Prentice-Hall, 1996.

[13] R. Scholtz, R. Weaver, E. Homier, J. Lee, P. Hilmes, A. Taha, and R. Wilson, "Ultrawideband radio deployment challenges," in Proc. Personal Indoor Mobile Radio Conf., London, U.K., Sept. 18-21, 2000.

[14] B. Razavi, RF Microelectronics. Englewood Cliffs, NJ: Prentice-Hall, 1997.

[15] P. P. Vaidyanathan, Multirate Systems and Filter Banks. Englewood Cliffs, NJ: Prentice-Hall, 1993.

[16] R. Cramer, "An evaluation of ultra-wideband propagation channels," Ph.D. dissertation, University of Southern California, Dec. 2000.

[17] N. Jayant and P. Noll, Digital Coding of Waveforms. Englewood Cliffs: Prentice-Hall, Inc., 1984.

[18] A. Papoulis, "Generalized sampling expansion," IEEE Trans. Circuits Syst., vol. 24, pp. 652-654, Nov. 1977.

[19] J. Cioffi, Data Signals, Sets, and Detection, ser. EE379a class notes. Stanford, CA: Stanford Univ. Press.

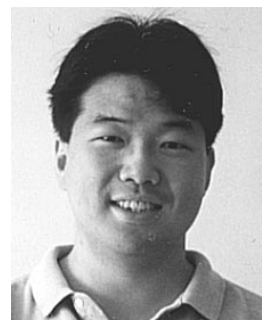

Won Namgoong (S'94-M'99) received the B.S. degree in electrical engineering and computer science from the University of California, Berkeley, in 1993, and the M.S. and Ph.D. degrees in electrical engineering from Stanford University, Stanford, CA, in 1995 and 1999, respectively.

In 1999, he joined the faculty of the Electrical Engineering Department, University of Southern California, Los Angeles, where he is an Assistant Professor. His current research activities include signal processing systems, wireless communication, and RF/mixed signal circuits and systems.

Dr. Namgoong received the National Science Foundation (NSF) CAREER Award in 2002 